\title{
Etude du cycle nycthéméral de la teneur en oxygène dissous en bassins de pisciculture par la méthode des moyennes mobiles. Relation avec la température et l'insolation Study of the daily fluctuations of dissolved oxygen in fish ponds by the moving-average method Relation with temperature and solar radiations
}

\author{
D. Barthelemy et M. Goubier
}

Volume 4, numéro 3, 1991

URI : https://id.erudit.org/iderudit/705107ar

DOI : https://doi.org/10.7202/705107ar

Aller au sommaire du numéro

Éditeur(s)

Université du Québec - INRS-Eau, Terre et Environnement (INRS-ETE)

ISSN

0992-7158 (imprimé)

1718-8598 (numérique)

Découvrir la revue

Citer cet article

Barthelemy, D. \& Goubier, M. (1991). Etude du cycle nycthéméral de la teneur en oxygène dissous en bassins de pisciculture par la méthode des moyennes mobiles. Relation avec la température et l'insolation. Revue des sciences de l'eau / Journal of Water Science, 4(3), 393-414. https://doi.org/10.7202/705107ar

\section{Résumé de l'article}

Dans ce travail, le cycle journalier de l'oxygène dissous en bassins de pisciculture semi-intensive a été étudié en relation avec des données météorologiques. Nous disposions pour cela de 22 semaines d'enregistrements (mesures horaires) de janvier 1988 à décembre 1989, dont une semaine de mesures sous glace. Chaque période de mesures a été traitée comme une série chronologique en pratiquant une décomposition saisonnière par la méthode des moyennes mobiles qui permet d'extraire à partir d'une série de mesures la tendance sur la période étudiée et la composante journalière.

Cette méthode, montre que le cycle nycthéméral de l'oxygène dissous garde son aspect et classique toute l'année, excepté sous la glace. Toutefois, l'amplitude journalière de la teneur en oxygène varie beaucoup selon la saison, et nous avons pu distinguer une période estivale où le métabolisme de l'étang semblait accéléré. L'analyse du cycle nycthéméral de l'oxygène dissous, dans lequel on distingue production et consommation, comparé à ceux de la température de l'eau et de l'insolation journalière moyenne, a permis d'étudier ce phénomène et de montrer l'importance des facteurs météorologiques dans la détermination du bilan de l'oxygène dissous en étang. 


\title{
Etude du cycle nycthéméral de la teneur en oxygène dissous en bassins de pisciculture par la méthode des moyennes mobiles Relation avec la température et l'insolation*
}

\author{
Study of the daily fluctuations of dissolved oxygen \\ in fish ponds by the moving-average method \\ Relation with temperature and solar radiations
}

D. BARTHELEMY1, M. GOUBIER 1,2

Reçu le 5 julliet 1990, accepté pour publication le 8 mars $1991^{\text {* * }}$.

RÉSUMÉ

Dans ce travail, le cycle journalier de l'oxygène dissous en bassins de pisciculture semi-intensive a été étudié en relation avec des données météorologiques. Nous disposions pour cela de 22 semaines d'enregistre ments (mesures horaires) de janvier 1988 a décembre 1989, dont une semaine de mesures sous glace. Chaque période de mesures a été traitée comme une série chronologique en pratiquant une décomposition saisonnière par la méthode des moyennes mobiles qui permet d'extraire à partir d'une série de mesures la tendance sur la période étudiée et la composante journafière.

Cette méthode, montre que le cycle nycthéméral de l'oxygène dissous garde son aspect « classique " toute l'année, excepté sous la glace. Touteiois, I'amplitude journalière de la teneur en oxygène varie beaucoup selon la saison, et nous avons pu distinguer une période estivale où le métaholisme de l'étang semblait accéléré. L'analyse du cycle nycthéméral de l'oxygène dissous, dans lequel on distingue production el consommation, comparé à ceux de la température de l'eau et de l'insolation journalière moyenne, a permis d'étudier ce phénomène et de montrer limportance des facteurs météorologiques dans la détermination du bilan de l'oxygène dissous en étang.

Mots clés : Oxygène, cycle nycthéméral, variations annuelles, étang, météorologie, décomposition saisonnière.

1. Institut Régional de Recherches appliquées en Aquaculture, Université Catholique de Lyon, 25, rue du Plat, 69288 Lyon cedex 02.

2. Université Jean Moulin, Lyon III, 1, nue de l'Université, 69007 Lyon.

- Communication présentée au 34 Congrés de l'Association Française de Limnologie, Metz-Nancy, 29-31 mai 1990.

* Les commentaires seront recus jusqu'au 30 mars 1992. 
Dissolved oxygen is a very important factor in fish ponds, particularly under intensive conditions. Thus, the daily fluctuations of dissolved oxygen have been studied in different countries and under different conditions (lakes, ponds, rivers), but with data series generally limited to a few measurements per day. The automatic data recorder of the IRRA'S experimental station provides very long series of data concerning of dissolved oxygen, temperature and meteorological factors through hourly measurements which allow the use of mathematical analysis like the moving average method. This automatic data recorder has been in use since September 1987 and the data obtained have been analysed in research on summer oxygen deficit prevention. After 3 years' measurements it was interesting to investigate the annual change in the daily fluctuations of the dissolved oxygen. For this, daily hourly measurements of dissolved oxygen, temperature and solar radiations were taken and examined during 22 weeks from January 1988 to December 1989 (one week under ice cover). Oxygen data were analysed using seasonal decomposition by the moving average method, and the results were compared with the temperature and solar radiation measurements.

The measurements were taken from 6 of the 16 IRRA'S experimental ponds (in the Dombes region, to the North-East of Lyon, France) and in a fish pond. Alt these ponds were semi-intensive fish-ponds stocked with Carp (Cyprinus carpio) Tench, (Tinca tinca), Grass Carp (Clenopharingodon idella) and Silver Carp (Hypophthalmichthys molitrix).

The seasonal decomposition through the moving average method devides the time series data into a trend (long term fluctuations), seasonal indices (or daily component) and residuals, but only seasonal indices, representing the daily variations, are studied here. The daily component was next split into oxygen production and consumption if respiration is taken to be constant over one day and can be evaluated through the night oxygen decrease (EOELINE ef al., 1968; ROMAIRE and BOYD, 1978; BARTHELEMY and CDUTUAE, 1990).

Through this method, it appeared that the daily fluctuations of dissolved oxygen had the same classical aspect all the year round, except under ice cover, where it was impossible to observe a significant oxygen production. Such fluctuations, which are the result of the effect of oxygen production and consumption, have also been observed under different conditions (BOYD and LICHTXOPPLER, 1979 ; SIN and CHIU, 1982 ; KEPENYES and VARADI, 1984 ; MARZOLF and SAUNDERS, 1984 ; GINOT, 1988 ; COUTURE, 1988). The aspect of the daily cycles was relatively constant but it was possible to distinguish 3 periods according to the amplitude of these cycles:

- a winter period with less than $2 \mathrm{mgh}$ daily variations,

- a summer period when these variations were greater than 4 or $7 \mathrm{mg} /$,

- an intermediate period in spring and autumn.

As these 3 periods did not occur at the same time of the year for $1988 \& 1989$, it appeared that the meteorological conditions (temperature and solar radiations) were important factors for determining these periods in the year.

The study of oxygen production and consumption gave the same conclusions.

These results have been compared with meteorological data : mean water temperature over 7-day periods (168 measurements), plus mean cumulative daily solar radiations over 7 days.

There appeared significant correlations between the oxygen production and the consumption, with these meteorological factors, but these relations were different for the " summer * period (June to October) and the rest of the year. There was a delay between the period when temperature and solar radiations 
are best for primary production (June and July) and the period when the pond's metabolism is maximum.

There have been no measurements on primary production or algal population dynamics to explain these results clearly, but considering the observations of GRYGIEREK and WASILEWSKA (1979) and ELORANTA (1985) it is possible to give an explanation. In May-June the ponds are characterized by clear water (a higher than $60 \mathrm{~cm}$ transparency was observed) with low density algal populations, thus the pond ecosystem is not ready to use thermic and light energy. This delay between meteorological conditions and oxygen dynamics have been observed by ELORANTA (1985) and BOYD (1985).

These results are useful in pond management, particularly when it is necessary to prevent oxygen depletion. The risk of oxygen deficits is greater in August-September, when the ecological conditions are very unstable owing to the important daily oxygen fluctuations. In this case, meteorological changes like temperature decreases or rain-tall can perturb the ecosystem and cause algal die-off with a great risk of oxygen depletion.

The aim of this study was to show the possibitities of seasonal decomposition by the moving average method in the analysis of oxygen measurements. When it is possible to get long serie data, with constant skip, this method is very valuable for studying long term and daily fluctuations. The decomposition of the daily component through the method of EOELINE et al. (1968) also gives indications on primary production and oxygen consumption.

Key-words : Oxygen, daily cycle, annual variations, fish-pond, meteorology, seasonal decomposition.

\section{1 - INTRODUCTION}

Depuis sa création en 1985, l'Institut Régional de Recherches appliquées en Aquaculture s'intéresse à l'étude du cycle de l'oxygène dissous en étang de pisciculture, avec pour objectif principal, la prévision des risques de désoxygénations estivales par la mise au point d'une méthode utilisable par les pisciculteurs.

Dans le cadre de ces travaux, et en association avec une étude en cours sur le climat de la Dombes, une station de mesures en continu de la température de l'eau, du taux d'oxygène dissous ainsi que des paramètres météorologiques a été mise en place. Malgré quelques aléas dans la mise au point de ce matériel (perturbations électriques, détérioration par des rats musqués), une quantité importante de données a pu être rassemblée depuis plus de deux ans. Nos objectifs de recherche prioritaires nous avaient jusqu'à présent conduits à nous intéresser essentiellement aux mesures effectuées en été, période pendant laquelle les risques de déficit en oxygène sont les plus importants, mais il nous a paru intéressant de dresser un bilan à l'échelle de lannée à partir des données déjà obtenues.

Le présent travail constitue une première partie de ce bilan qui devrait permettre d'observer l'évolution annuelle du cycle nycthéméral de l'oxygène dissous en relation avec quelques facteurs météorologiques. 
Les données exploitées ici n'ont pas été récoltées initialement dans le but d'une étude du cycle annuel de l'oxygène, mais dans le cadre d'expériences à plus court terme n'incluant pas de mesures de productivité primaire ou d'étude des populations algales. II en résulte une certaine hétérogénéité (différents sites étudiés) et un manque de précision, mais nous allons voir qu'il est possible, à l'aide de méthodes adaptées, d'en dégager des phénomènes globaux significatifs.

L'évolution annuelle du cycle nycthéméral de l'oxygène dissous sera d'abord étudiée, nous analyserons ensuite les relations existant entre l'insolation, la production et la consommation d'oxygène.

\section{2 - MATÉRIEL ET MÉTHODES}

\subsection{Site}

Ce travail a été réalisé sur la station expérimentale de l'I.R.R.A. à SaintNizier-le-Désert (Ain), composée de 16 bassins en terre, d'une profondeur moyenne de $1 \mathrm{~m}$ à $1,20 \mathrm{~m}$ pour une surface de 500 à $1400 \mathrm{~m}^{2}$, encadrés par deux étangs de pisciculture (altitude de la station : $250 \mathrm{~m}$ ). Ces bassins sont utilisés pour des expérimentations sur la croissance des poissons en conditions d'élevage semi-intensives proches de celles pratiquées en Dombes (les rendements nets au moment des pêches sont de l'ordre de 300 à $600 \mathrm{~kg} / \mathrm{ha}$ en moyenne). Les espèces maintenues en élevage sont essentieltement la Carpe commune (Cyprinus carpio), la Tanche (Tinca tinca) et les Carpes herbivores (Ctenopharingodon idella) et argentées (Hypophthalmichthys molitrix).

\subsection{Paramètres mesurés}

Sur le site décrit plus haut, ont été mesurés :

- la température de l'air et le rayonnement solaire global (watts $/ \mathrm{m}^{2}$ ),

- la température de l'eau et la teneur en oxygène dissous à une profondeur de $20 \mathrm{~cm}$, sur 6 bassins différents et un des deux étangs adjacents à la station. Les conditions d'élevage dans ces 7 sites sont voisines, mais il est clair que les conditions écologiques peuvent varier de façon notable (notamment du fait de la diversité des sédiments), ce qui entraîne une variabilité limitant la généralisation des résultats.

\subsection{Matériel de mesures}

Il est en place sur la station depuis fin 1987 et est composé :

- des capteurs : sonde Heito pour la température de l'eau et l'oxygène dissous, girouette, anémomètre et sonde thermique Jules Richard Instruments, et pyranomètre Cimel Electronique E80 ; 
- de la centrale de mesure et d'enregistrement Auteg Emac85 qui permet d'enregistrer les valeurs des paramètres ci-dessus selon un pas de temps compris entre 3 et 60 minutes (dans notre cas : $60^{\circ}$ ).

Les résultats des mesures sont stockés dans la centrale et peuvent être récoltés à distance grâce à un modem téléphonique. Les mesures de température et d'oxygène dissous enregistrées, représentent une valeur instantanée au moment du réveil de la centrale, mais pour l'insolation (ou radiation solaire), il s'agit de la somme des radiations reçues pendant l'heure précédant la mesure. L'heure donnée par la centrale est l'heure solaire (T.U.).

\subsection{Calendrier des mesures retenues}

La mesure des paramètres météorologiques n'a pas posé de problèmes, à part quelques rares pannes. Par contre, pour la mesure de l'oxygène dissous, la mise au point du matériel, les problèmes d'étalonnage et de détérioration par les rats musqués ont occasionné des lacunes dans les séries de données. L'étalonnage des sondes à oxygène était effectué à l'air (corrigé en fonction de l'altitude et non de la pression atmosphérique) pour une large gamme de température. Cette méthode permettait de réaliser une compensation précise de la mesure en fonction de la température et donc de réduire la fréquence des étalonnages. Les sondes étaient nettoyées toutes les semaines en été et 1 à 2 fois par mois en hiver.

Le fait de ne pas tenir compte de la pression atmosphérique peut sembler a priori être une source d'erreur ou d'incertitude, mais les variations de ce paramètre pour un site donné ont un impact assez faible sur la solubilité de l'oxygène dans l'eau. On peut citer comme exemple les valeurs de pression atmosphérique dans le département de l'Ain à une altitude de $250 \mathrm{~m}$ (station d'Ambérieu, appartenant au réseau de la Météorologie Nationale) entre le $01 / 07 / 87$ ét le 30/09/87 qui étaient comprises entre 971 et $995 \mathrm{hPa}$. L'utilisation de l'altitude comme facteur de correction a donné par rapport à ces valeurs extrêmes des erreurs de seulement $+7 \%$ et $-1 \%$ dans la détermination de la concentration standard en oxygène.

Sur l'ensemble des mesures effectuées depuis 2 ans, on a sélectionné des périodes de 7 jours consécutifs par mois, pour lesquels les enregistrements de la teneur en oxygène dissous étaient de bonne qualité. Le présent travail a été réalisé à partir de 22 semaines de mesures (tableau 1), dont une semaine de gel continu (début décembre 1989).

\subsection{Décomposition saisonnière par la méthode des moyennes mobiles}

Les données récoltées constituent des séries chronologiques, c'est-à-dire une succession de mesures effectuées à intervalle régulier, dont linterprétation peut être réalisée au moyen de méthodes mathématiques adaptées, comme la décomposition saisonnière par la méthode des moyennes mobiles. Cette méthode est très intéressante pour l'étude des phénomènes saisonniers (ou journaliers), mais son application en écologie est assez rare, car elle 
Tableau 1 Date des périodes d'enregistrement étudiées.

Table 1 Dates of studied measurements.

\begin{tabular}{|c|c|c|c|}
\hline \multicolumn{2}{|c|}{1988} & \multicolumn{2}{|c|}{1989} \\
\hline dates & $n^{\circ}$ semaine & dates & $n^{\circ}$ semalne \\
\hline $\begin{array}{c}\text { du } 7 \text { au } 13 / 1 \\
\text { du } 12 \text { au } 18 / 2 \\
\text { du } 24 \text { au } 30 / 4 \\
\text { du } 13 \text { au } 19 / 5 \\
\text { du } 18 \text { au } 24 / 6 \\
\text { du } 18 \text { au } 24 / 7 \\
\text { du } 13 \text { au } 19 / 8 \\
\text { du } 21 \text { au } 27 / 9 \\
\text { du } 20 \text { au } 26 / 10 \\
\text { du } 24 \text { au } 30 / 12 \\
- \\
-\end{array}$ & $\begin{array}{l}2 \\
7 \\
17 \\
20 \\
25 \\
29 \\
33 \\
38 \\
42 \\
52\end{array}$ & $\begin{array}{c}\text { du } 16 \text { au } 22 / 1 \\
\text { du } 16 \text { au } 22 / 2 \\
\text { du } 30 / 3 \text { au } 05 / 4 \\
\text { du } 16 \text { au } 22 / 4 \\
\text { du } 19 \text { au } 25 / 5 \\
\text { du } 13 \text { au } 19 / 6 \\
\text { du } 25 \text { au } 31 / 7 \\
\text { du } 21 \text { au } 27 / 8 \\
\text { du } 18 \text { au } 24 / 10 \\
\text { du } 5 \text { au } 11 / 11 \\
\text { du } 4 \text { au } 10 / 12 \\
\text { du } 18 \text { au } 24 / 12\end{array}$ & $\begin{array}{c}3 \\
7 \\
13 \\
16 \\
21 \\
24 \\
30 \\
34 \\
42 \\
45 \\
49 \\
51\end{array}$ \\
\hline
\end{tabular}

nécessite des séries de données importantes. L'utilisation d'une centrale de mesure en continu est donc un atout important dans l'étude d'un cycle journalier comme celui de l'oxygène dissous. Cette méthode sera présentée en prenant comme exemple des enregistrements d'oxygène dissous réalisés en avril 1989.

A partir de l'examen de ces enregistrements (fig. 1a), on distingue aisément que les données brutes peuvent être la résultante de deux composantes:

- une composante cyclique (période : 24 heures) qui sera appelée saisonnalité ou composante journalière (dans le cas d'un cycle journalier),

- une composante globale qui reflète l'évolution générale du paramètre, on parlera de tendance.

Le but de la décomposition saisonnière est de séparer ces deux composantes afin de faire la part des variations journalières et des variations à plus long terme.

Pour réaliser cette décomposition, on considére que les valeurs mesurées $X_{t}$ peuvent être exprimées par l'équation suivante (GOURIEROUX et MONFORT, 1977) :

$X_{t}=Z_{t}+S_{t}+u_{t}$

ou :

$-Z_{t}$ est la tendance,

- $S_{t}$ la saisonnalité,

- et $u_{t}$ le résidu qui est une composante aléatoire indépendante du phénomène cyclique observé.

Cette décomposition journalière est généralement réalisée à l'aide de logiciels adaptés, mais son principe est relativement simple.

On réalise d'abord une moyenne mobile dont le terme est égal à la longueur de la saisonnalité que l'on veut étudier (24 dans notre cas). 
La série de valeurs obtenue est plus courte de 24 mesures que la série initiale mais on constate la disparition des variations cycliques. Ces moyennes mobiles constituent la tendance (fig. 1a). Lorsqu'on fait la différence entre les valeurs initiales et les moyennes mobiles $\left(X_{t}-Z_{t}\right)$, on ne conserve que les variations journalières à partir desquelles on peut faire les moyennes horaires qui nous donnent la composante journalière (fig. 1b). Il est alors aisé, à partir de l'équation (1), d'en déduire les résidus (fig. 1c).

$\mathrm{mg} / \mathrm{l}$ oxygène
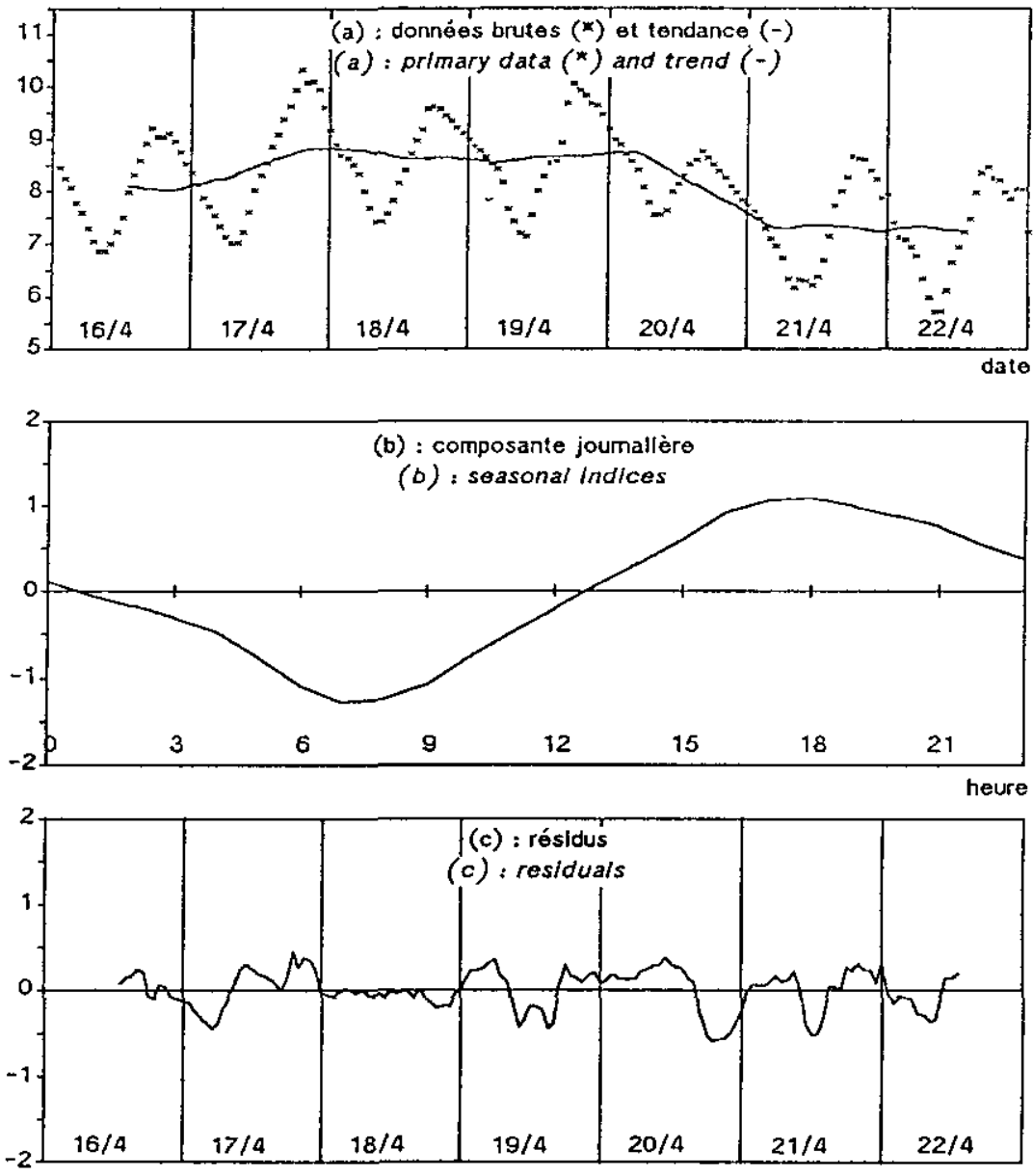

date

Figure 1 Décomposition saisonnière d'une série chronologique, application à des mesures d'oxygène dissous.

Seasonal decomposition of time series data, application to dissolved oxygen measurements. 
La composante journalière et les résidus sont représentés par des valeurs positives et négatives qui expriment les variations du paramètre autour de la tendance, ils ont donc la même unité que les mesures. Les résidus sont un " bruit de fond " indépendant des variations journalières. Malgré leur nom à consonance péjorative, ils peuvent constituer un élément important dans l'interprétation des données. Ainsi, leur comparaison à la saisonnalité peut donner une indication sur la qualité de cette dernière.

Le travail qui suit, ne concerne que l'analyse des composantes journalières, les résidus ne seront pris en compte que lorsque la composante journalière semblera discutable. Afin de limiter les risques d'erreur, nous avons choisi des périodes de mesures pour lesquelles les variations de la tendance étaient faibles, de façon à mieux appréhender le cycle nychtéméral de l'oxygène dissous.

\section{3 - RAPPELS SUR LE CYCLE NYCTHÉMÉRAL DE L'OXYGÉNE DISSOUS}

Les variations nycthémérales de la teneur en Oxygène dissous en étang, ont pratiquement toujours le même aspect, quel que soit le climat ou le type de pisciculture (BOYD et LICHTKOPPLER, 1979 ; SIN et CHIU, 1982 ; KEPENYES et VARADI, 1984 ; MARZOLF et SAUNDERS, 1984 ; GINOT, 1988 ; COUTURE, 1988). cette évolution est essentiellement la résultante de l'équilibre entre production et consommation d'oxygène, et les variations journalières ont l'aspect de celles présentes sur la figure $1 \mathrm{~b}$. La distinction de la part des différentes composantes du bilan de l'oxygène dissous est difficile, voire impossible (LINGEMAN, 1980) et EDELINE et al. (1968) proposent une méthode simple et approchée pour séparer production et consommation (fig. 2).

La consommation globale d'oxygène est appréhendée par l'examen de la phase nocturne du cycle de l'oxygène. Cette partie de la courbe peut être assimilée à une droite (ROMAIRE et BOYD, 1978 ; BARTHELEMY et COUTURE, 1990) dont la pente représente l'intensité du phénomène. Considérant la consommation comme constante au cours d'une journée, on peut extrapoler l'évolution de la teneur en oxygène en absence de production.

En faisant la somme entre la teneur mesurée en oxygène et la droite de consommation, on obtient une courbe d'aspect sigmoïde qui représente le cumul de la production d'oxygène.

Cette méthode est proche de celle proposée par McCONNEL (1962; in OLAH, et al., 1978) et de celle utilisée par LINGEMAN (1980). Une telle approche est simple à réaliser et peut fournir des informations intéressantes, mais elie est entachée d'erreur dans la mesure où elle considère la consommation d'oxygène par le milieu comme constante. En effet, la « respiration n d'un étang n'est pas un phénomène constant sur la journée, elle est généralement plus 


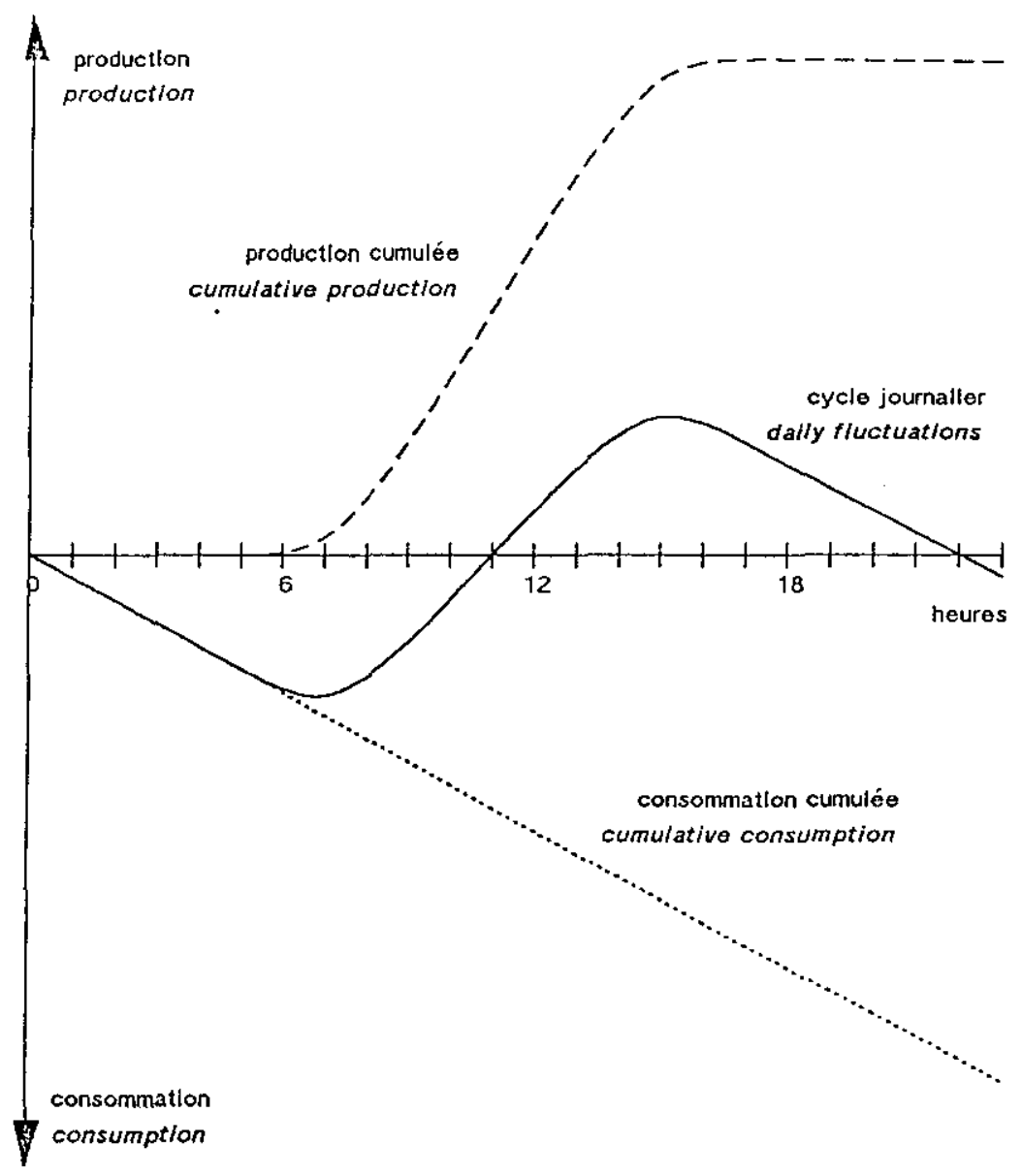

Figure 2 Décomposition schématique du cycle journalier de l'oxygène dissous (d'après Edeline et al., 1968).

Schematic decomposition of daily fluctuations of dissolved oxygen, (in Edeline et al., 1968).

élevée l'après-midi (LINGEMAN, 1980). Ceci vient du fait que la respiration du milieu (dans des bassins de pisciculture) augmente avec la teneur en oxygène dissous (OLAH et al., 1978). L'erreur sera donc théoriquement d'autant plus élevée que les variations de la teneur en oxygène seront importantes. Toutefois, le fait que la baisse nocturne de la teneur en oxygène soit linéaire (corrélation hautement significative, BARTHELEMY et COUTURE, 1990) suggère que dans notre cas la respiration est peu dépendante de l'oxygénation du milieu et qu'elle peut être considérée comme constante (au moins pendant la nuit). 
La description ci-dessus des caractéristiques d'un cycle type s'applique aux mesures brutes de la teneur en oxygène, mais peut être également utilisée pour étudier des composantes journalières, ce qui sera fait dans la suite de ce travail.

\section{4 - RÉSULTATS}

\subsection{Evolution annuelle de la composante journalière}

La figure 3 montre pour les deux années d'étude l'évolution annuelle de la composante journalière de l'oxygène dissous. Celle-ci a pratiquement le même aspect tout au long de l'année, excepté pour les mesures effectuées sous glace (début décembre 1989). Dans ce cas, l'aspect de la composante journalière est très altéré, et une anayse des résidus montre qu'ils ont des valeurs supérieures aux coefficients saisonniers (fig. 4), ce qui traduit l'absence, dans les conditions de cette étude, d'un cycle journalier marqué, donc de production primaire sous la glace. Ainsi, lorsque la couverture glacée se prolonge, il peut y avoir épuisement du milieu en oxygène (BARICA et MATHIAS, 1979 ; MATHIAS et BARICA, 1980). Par contre, en ce qui concerne l'amplitude des variations journalières on observe, comme SIN et CHIU (1982), des différences inter-saisons très marquées. Les mois d'août, septembre, octobre 1988, et de juin, juillet, août 1989, sont caractérisés par des variations journalières très importantes. La figure 5 montre de façon plus précise l'évolution de la composante journalière, et notamment de son amplitude. On peut distinguer 3 périodes :

- une période hivernale où l'amplitude des variations journalières est inférieure à $2 \mathrm{mg} / \mathrm{l}$,

- une période estivale pour laquelle ces variations dépassent 4 et même $7 \mathrm{mg} / \mathrm{l}$,

- une période intermédiaire au printemps et en automne.

Le positionnement de ces périodes ne semble pas fixé de façon précise d'une année à l'autre ; c'est ainsi que la période " estivale " est plus précoce en 1989. Par contre, les variations journalières sont identiques en janvier et février 1988 et 1989 . Toutefois, l'absence de continuité dans les séries de mesures ne permet pas de comparer exactement les deux années d'étude.

\subsection{Analyse des variations journalières}

Une étude de la composante journalière, selon la méthode proposée par EDELINE et al. (1968), permet de séparer la consommation de la production d'oxygène. Dans le cadre de cette étude, une telle analyse doit être effectuée avec prudence dans la mesure où elle part d'une hypothèse de travail qui n'est pas vérifiée par une expérimentation propre (mesure de production primaire par exemple), et où elle est effectuée à partir de moyennes. 


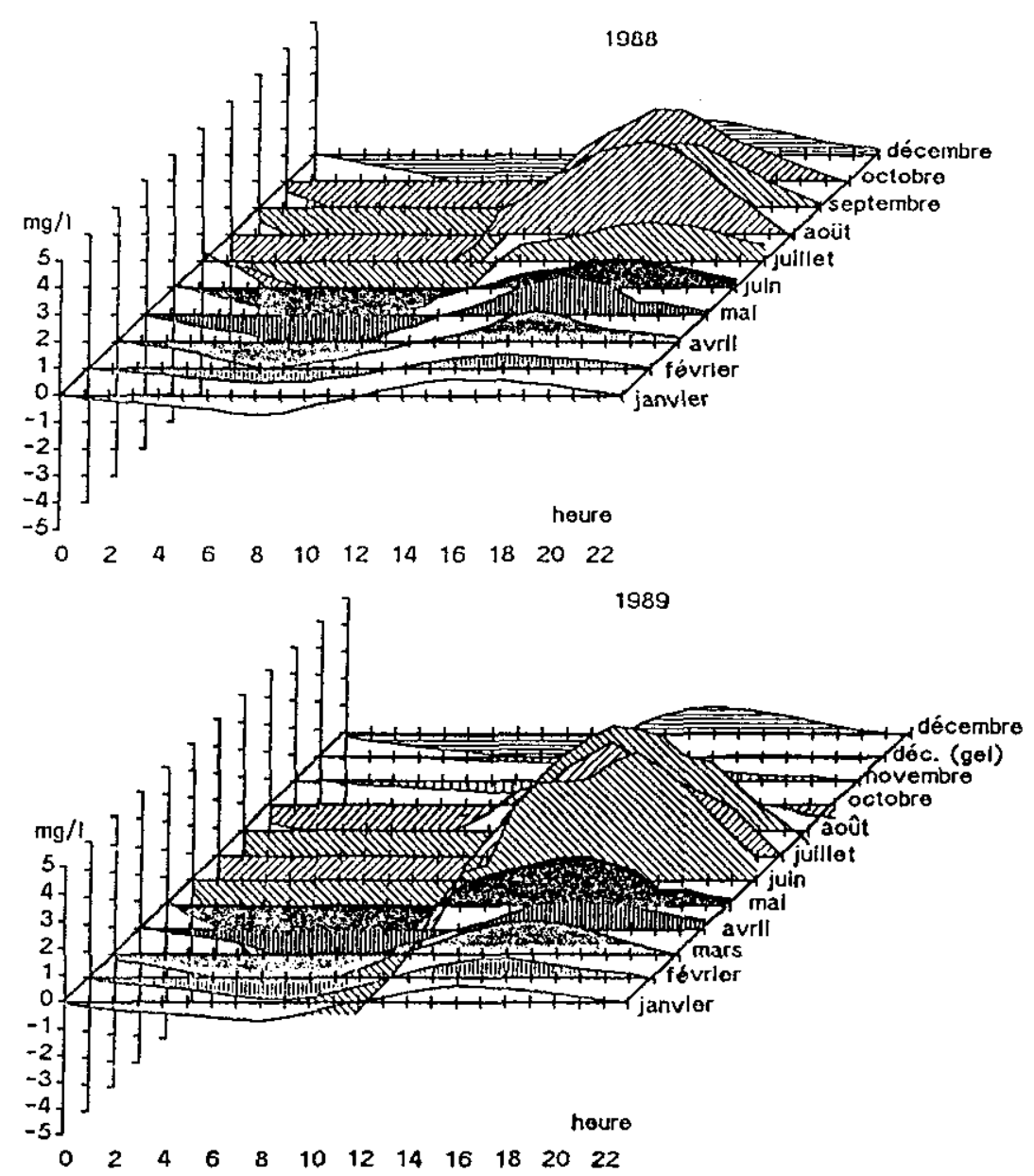

Figure 3 Evolution annuelle de la composante journalière de l'oxygène dissous. Annual evolution of seasonal indices of dissolved oxygen.

\subsubsection{Consommation d'oxygène}

La figure 6 représente l'évolution de la pente (en $\mathrm{mg} / \mathrm{h} / \mathrm{h}$ ) de la consommation nocturne au cours des deux années d'étude. La courbe obtenue a un aspect proche de celle de lamplitude des coefficients saisonniers, on note en effet les consommations les plus importantes en août, septembre, octobre 1988, et juin, juillet, août 1989. Cette consommation représente l'activité respiratoire du plancton, du sédiment et des poissons ainsi que les pertes dues à la diffusion dans l'air en cas de sursaturation. Les chiffres obtenus, entre 0,1 et $0,4 \mathrm{mg} / / \mathrm{h}$ pour la plupart, sont donc un peu supérieurs, tout en restant cohérents avec ceux de la littérature concernant la respiration de leau et du plancton (KEPENYES et VARADI, 1984 ; SCHROEDER, 1975 ; BOYD, 1982, 1985 ; BOYD et al., 1978). 

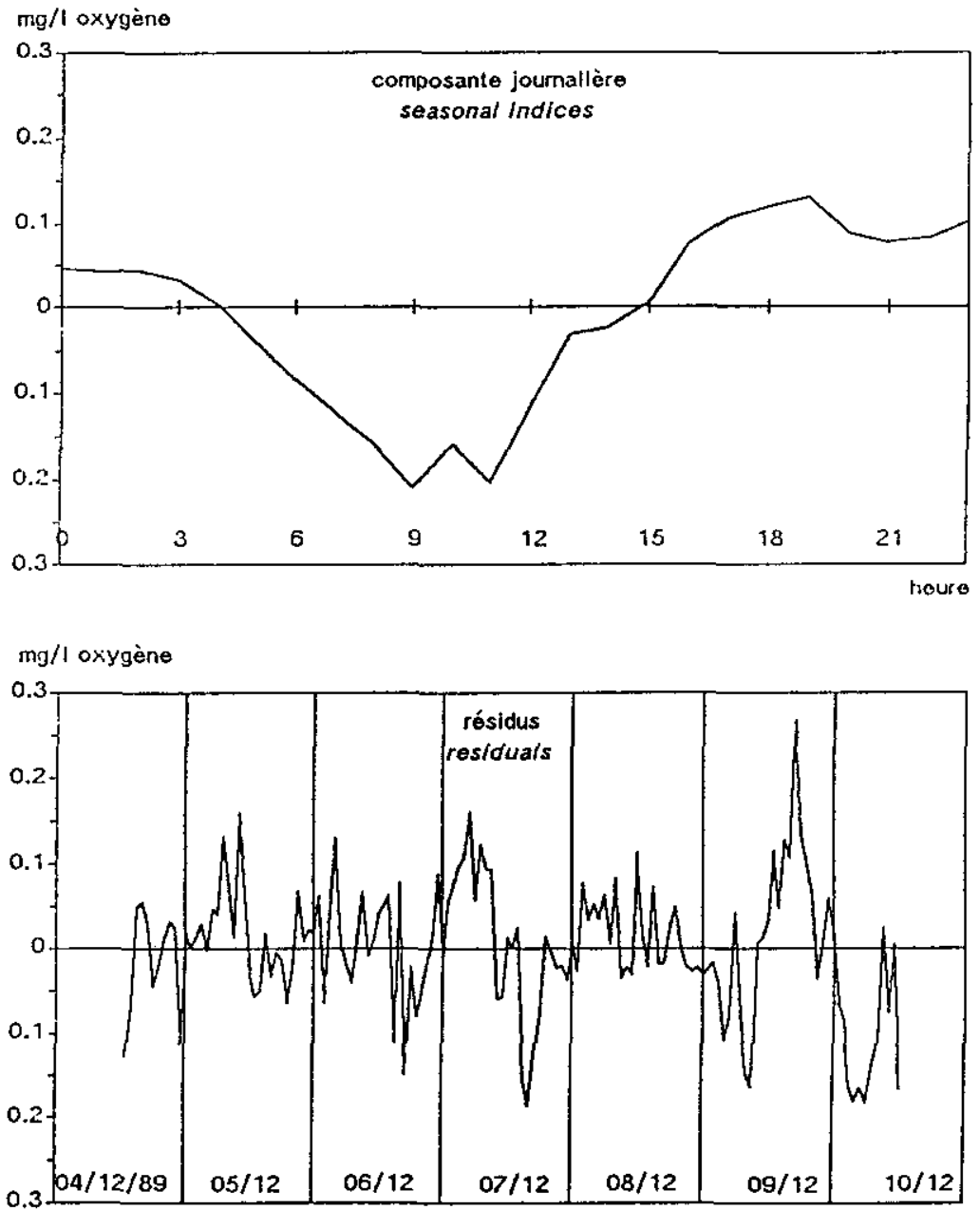

Figure 4 Suivi d'oxygène sous glace : composante saisonnière et résidus.

Oxygen measurements under ice cover : seasonal indices and residuals.

\subsubsection{Production d'oxygène}

Le graphique de l'évolution de la production journalière cumulée (fig. 7) donne exactement le même résultat que pour la consommation, avec des valeurs comprises entre 2 et $8 \mathrm{mg} / \mathrm{/j}$. Dans ce cas également ces données sont en accord avec celles généralement citées pour la production primaire en étang de pisciculture (SCHROEDER et BERNER-SAMSONOV, 1986 ; BOYD, 1982 ; COUTURE, 1988), mais une comparaison précise n'est pas possible dans la mesure où les conditions expérimentales sont mal connues ou trop différentes (climat, type d'exploitation, etc.). 


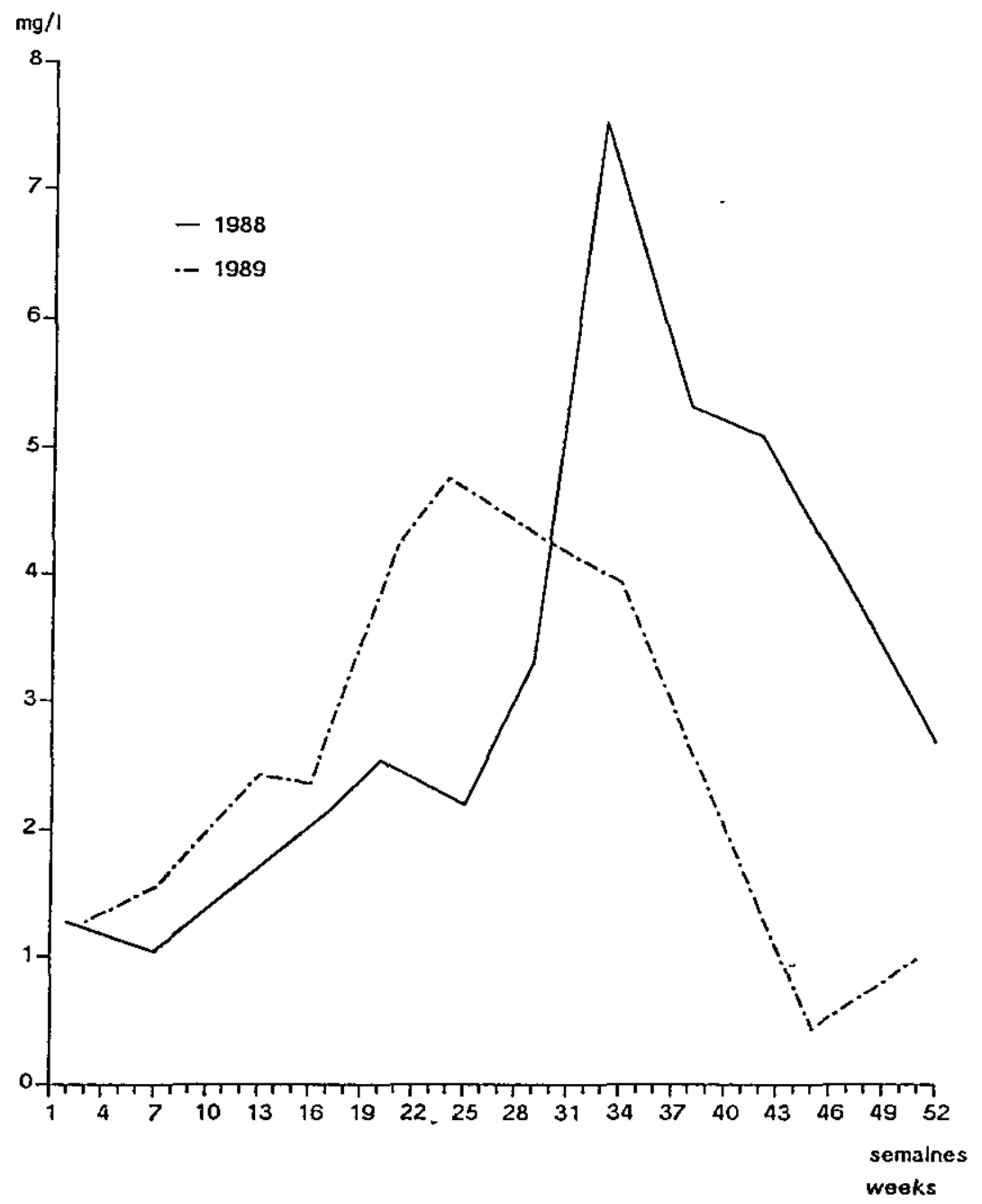

Figure 5 Evolution de ramplitude des coefticients journaliers en 1988 et 1989. Annual evolution of amplitude of seasonal indices, in 1988 and 1989. 


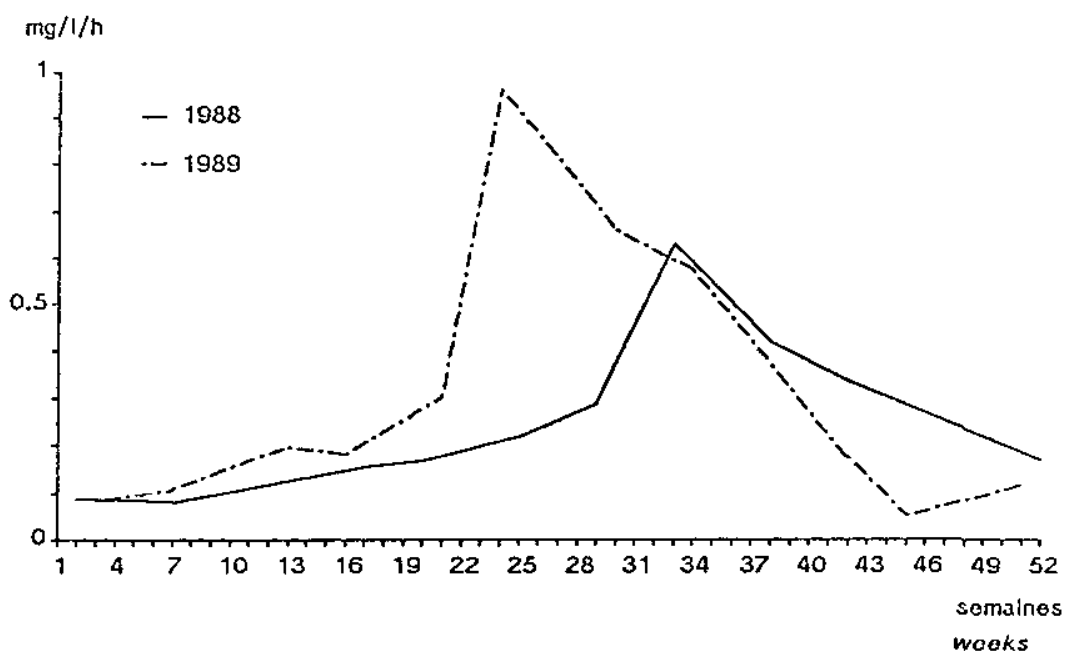

Flgure 6 Evolution de la consommation d'oxygène.

Evolution of the oxygen consumption.

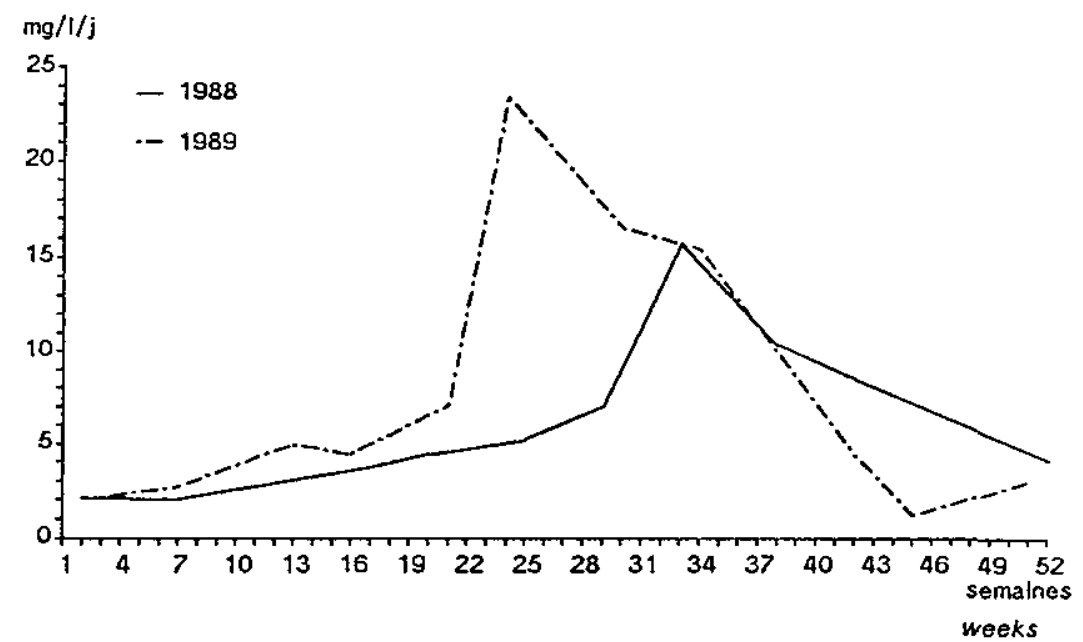

Figure 7 Evolution de la production journalière ơoxygène.

Evolution of the daily oxygen production. 


\subsection{Conclusion}

L'étude du cycle nycthéméral de l'oxygène dissous étang, en « dissociant" consommation et production journalière d'oxygène, a montré qu'au cours de la phase estivale le métabolisme de létang était "accéléré " avec une forte production, mais également une consommation importante.

Ces phénomènes sont en relation avec l'activité phytoplanctonique et les conditions climatiques, notamment l'insolation et la température de l'eau. Nous ne disposons pas d'étude des populations algales correspondant aux sites et périodes étudiées, mais nous avons par contre la mesure des paramètres métérologiques heure par heure. Il peut ainsi être intéressant de comparer ces données aux mesures d'oxygène dissous.

\section{5 - DISCUSSION : INFLUENCE DE CERTAINS FACTEURS CLIMATIQUES}

\subsection{Introduction}

Les facteurs climatiques tes plus importants intervenant dans l'évolution des variations nycthémérales de l'oxygène dissous sont la température de l'eau et l'insolation. Leur action sur le bilan de loxygène est complexe car elle intervient à plusieurs niveaux:

- la température influence la solubilité de l'oxygène dans l'eau et l'intensité des réactions métaboliques,

- le rayonnement solaire et la température de l'eau ont un rôle important sur l'activité photosynthétique et le développement des populations algales.

\subsection{Comparaison des conditions climatiques de 1988 et 1989}

Les données météorologiques utilisées ici sont celles fournies par la centrale de mesure aux mêmes périodes que celles définies pour l'oxygène (cf. 2.4). Nous utiliserons dans ce qui suit : les radiations journalières moyennes pour chaque période de 7 jours (soit 168 mesures), et la température moyenne (sur 7 jours également).

\section{Insolation}

La figure 8 montre pour 1988 et 1989 l'évolution des radiations jour nalières moyennes. On note que la période de juin à août présente des conditions identiques pour ces deux années, mais au mois de mai 89 les étangs ont reçu deux fois plus de radiations qu'en mai 88 . Ceci traduit sans doute une très faible nébulosité en mai 89 dont les valeurs d'insolation sont supérieures à celles de juin des deux années, sachant que c'est au solstice d'été (21 juin) que la durée théorique d'ensoleillement est maximum. 


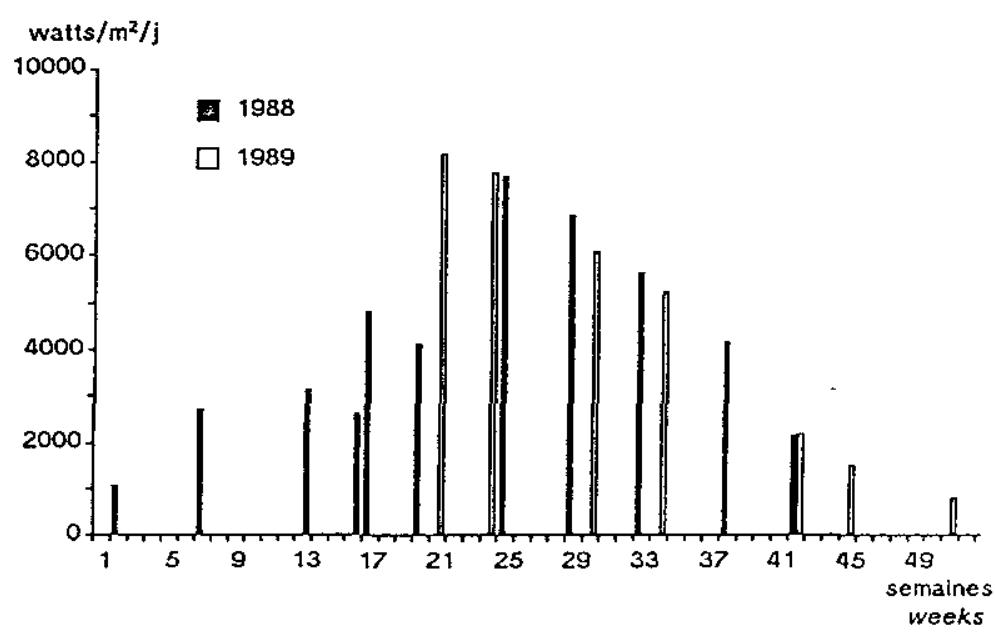

Figure 8 Evolution des radiations journalières moyennes.

Evolution of daily mean solar radiations.

\section{Température}

Les températures de l'air et de l'eau étant très corrélées $(r=0,963)$, seule cette dernière sera retenue. La figure 9 montre que pour les mois de mai et juin la température est plus élevée de $2^{\circ}$ à $4^{\circ} \mathrm{C}$ en 1989 qu'en 1988 . On note par contre le phénomène inverse en juillet et aôut. Ceci est analogue à ce que l'on observe pour l'insolation, mais les maximums de température sont décalés dans le temps par rapport à ceux de l'insolation.

Il apparaît donc, d'après ces deux paramètres, que les conditions théoriquement les plus favorables à une activité biologique et phytoplanctonique (lumière et température élevées) apparaissent dès le mois de mai en 1989 , mais seulement au mois de juin en 1988, et se prolongent jusqu'en septembre. Le rôle de ces facteurs climatiques dans le métabolisme d'étang est évident et il nous a paru intéressant de les comparer aux valeurs de consommation et de production calculées plus haut.

\subsection{Relation avec les facteurs météorologiques retenus}

Les variations de la consommation d'oxygène (pente de la droite et de la production journalière sont très corrélées $(r=0,999)$, ce qui est logique dans la mesure où les composantes journalières d'où eltes sont extraites ont des allures identiques ; leurs relations avec les facteurs climatiques seront donc similaires. 


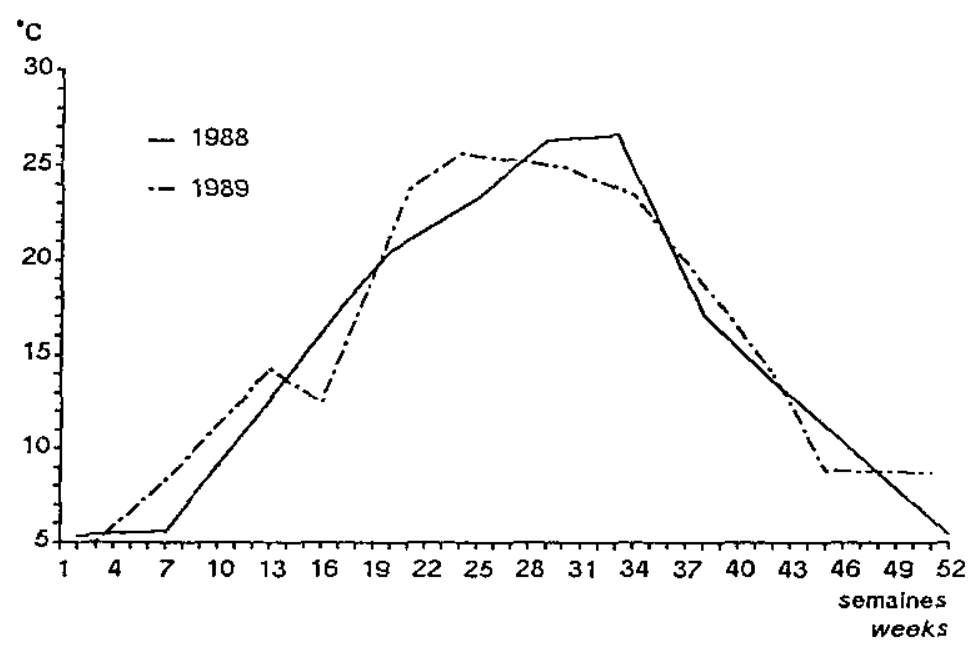

Figure 9 Evolution de la température moyenne de l'eau.

Evolution of the water mean temperature.

En ce qui concerne la relation entre la production, la consommation d'oxygène et l'insolation (fig. 10), on peut distinguer deux groupes de mesures : celles correspondant aux périodes estivales définies plus haut (août, septembre, octobre 1988 et juin, juillet, août 1989), et le reste des données. Pour chacun de ces groupes il existe une relation linéaire significative (au seuil de $1 \%$ ) avec l'ensoleillement journalier moyen. Vis-à-vis de la température de l'eau (fig. 11), on a exactement la même situation, à la différence près que juin 89 est en dehors des deux groupes cités plus haut.

Ce résultat tendrait à montrer que le fonctionnement des sites étudiés pourrait se faire selon deux allures :

- lorsque l'insolation et la température sont faibles à modérées, le métabolisme de l'étang est ralenti, avec un faible développement phytoplanctonique ;

- le passage à des conditions estivales conduit à un développement du phytoplancton responsable d'un accroissement du métabolisme de l'étang. Outre l'effet de la température sur le métabolisme des êtres vivants, le développement du phytoplancton va accroitte l'activité des différents compartiments : augmentation de la biomasse zooplanctonique et donc des poissons, stimulation de l'activité bactérienne minéralisatrice, tout ceci contribuant à augmenter la respiration du milieu. 

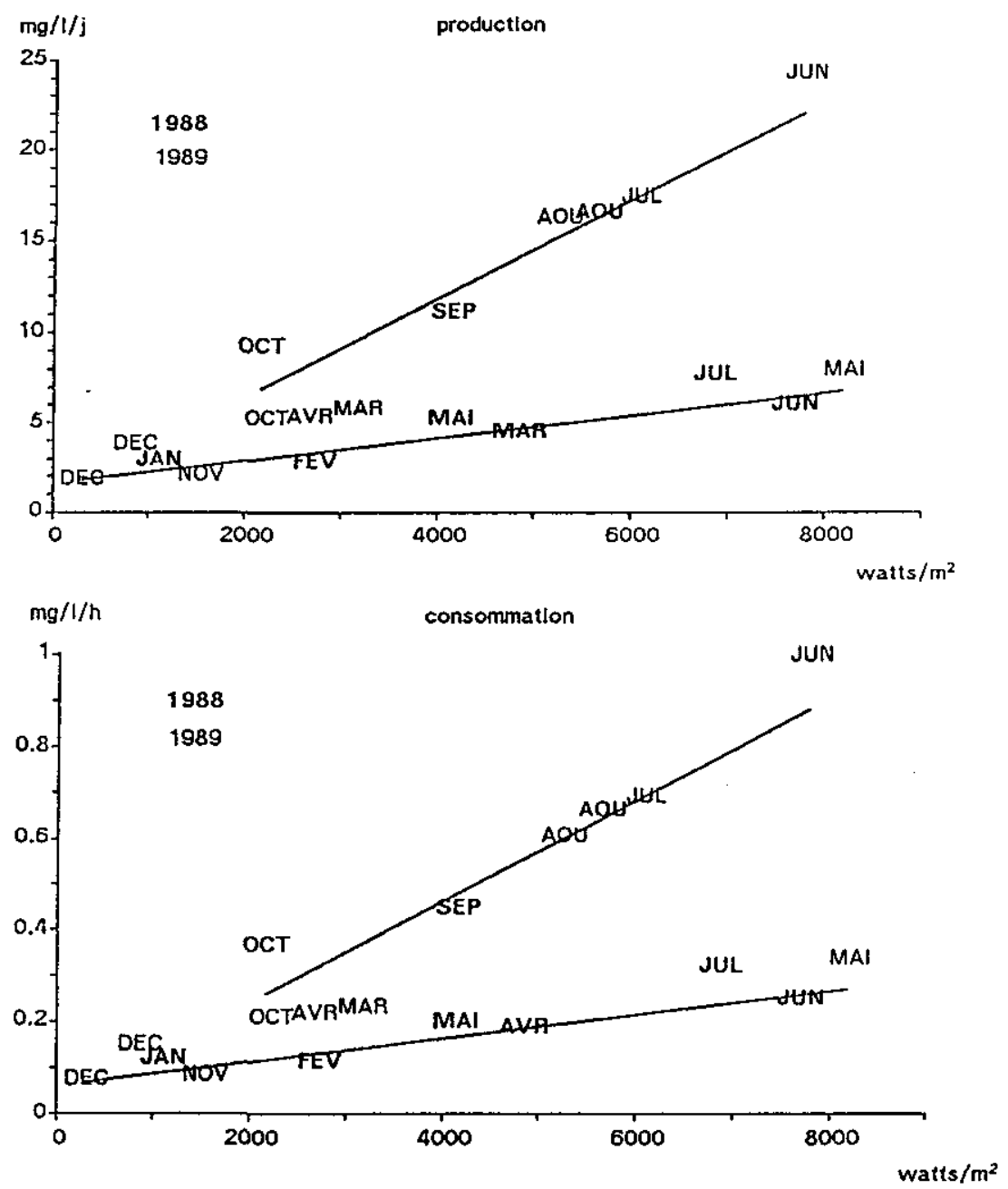

Figure 10 Relation entre la production et la consommation d'oxygène et les radiations joumalieres moyennes.

Relation between the oxygen production and consumption with the dailymean solar.

La distinction de ces deux périodes peut également être considérée comme étant le reflet d'une différence entre les cycles annuels de l'insolation et la température de l'eau, et ceux de la production et de la consommation d'oxygène. Les cycles des paramètres climatiques sont symétriques de part et d'autre d'un maximum qui est en mai/juin pour l'insolation et en juillet/août pour la température de l'eau, alors que ceux de la production et de la consommation d'oxygène sont nettement dissymétriques (fig. 6 et 7) avec un maximum en août/septembre. Il y a donc un décalage entre le moment où 

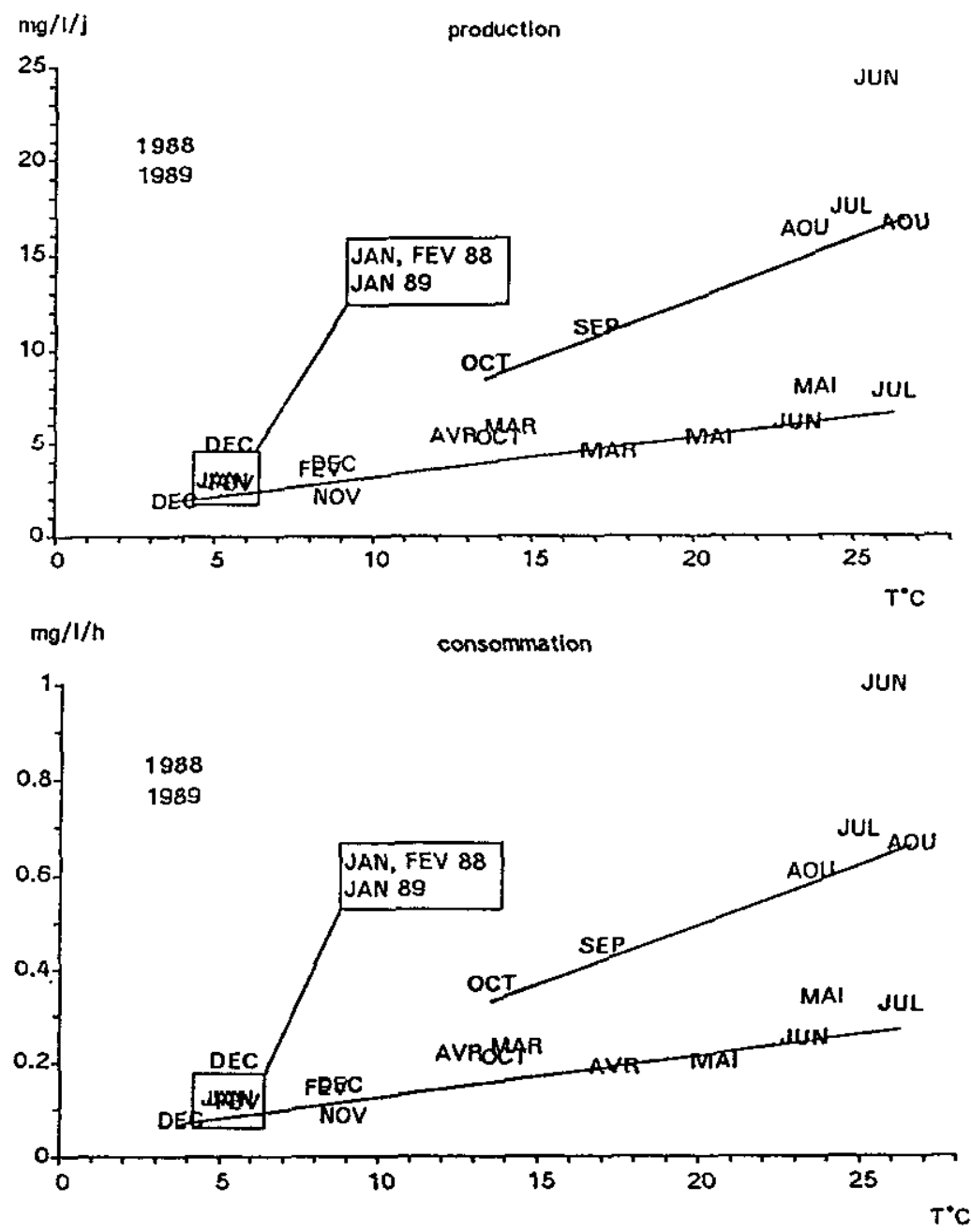

Flgure 11 Relation entre la production et la consommation o'oxygène et la température moyenne de l'eau.

Relation between the oxygen production and consumption with the water mean temperature.

l'insolation et la température sont maximum, et celui où les variations journalières de l'oxygène dissous sont les plus importantes. Ceci peut s'expliquer par le fait qu'en mai/juin la plupart des étangs sont en o phase claire " (GRYGIEREK et WASILEWSKA, 1979 ; ELORANTA, 1985) avec des eaux très pauvres en phytoplancton du tait du développement important des organismes zooplanc- 
toniques. Nous avons pu le constater sur différents bassins de notre station expérimentale en relevant des valeurs élevées de la transparence (supérieures à $60 \mathrm{~cm}$ ) jusqu'à début juillet pour les deux années. Ainsi, bien que les conditions soient favorables à l'activité phytoplanctonique, le milieu n'est pas " prêt " à exploiter les ressources lumineuses et caloriques. C'est après cette période que le phytoplancton se développe pour atteindre une activité maximale en août/septembre. L'observation de ce décalage rejoint les conclusions de LINGEMAN (1980) qui observe révolution cyclique annuelle (d'aspect sinusoïdal) de l'insolation, de la température, de la production et de la consommation d'oxygène. II note que production et consommation sont " en phase " et que le maximum de production d'oxygène se situe 2 à 3 semaines après le solstice d'été. ELORANTA (1985) observe également, sur des étangs eutrophes en Finlande, un décalage entre l'insolation maximum et les concentrations les plus élevées en chlorophylle qui sont observées au printemps et en automne. BOYD (1985) note aussi un maximum de production primaire et de respiration en août-septembre.

Des relations décrites plus haut, il n'est pas possible de dégager un effet de photoinhibition souvent mentionné dans la littérature (HUNDING, 1973 ; PEETERS et EILERS, 1978 ; PELLETIER, 1983). Cela vient sans doute du fait qu'il s'agit d'un phénomène ponctuel au cours de la journée qui est "gommé " par l'utilisation de moyennes et de productions journalières ; il est probable qu'à l'échelle annuelle son rôle dans le bilan de l'oxygène soit secondaire.

\section{6 - CONCLUSION GÉNÉRALE}

La mesure et l'enregistrement en continu de l'oxygène dissous et de quelques paramètres météorologiques, a permis d'acquérir une grande quantité de données, qui ont été dépouillées par la méthode des moyennes mobiles. Cette méthode, a permis de dégager, à partir des séries chronologiques, le cycle nycthéméral de l'oxygène dissous qui a été ensuite « dissocié " en production et consommation selon la méthode proposée par EDELINE et al. (1968). L'utilisation de ces méthodes (relativement simples) a fourni des éléments intéressants dans la compréhension du cycle annuel de l'oxygène dissous. Il est ainsi apparu, en accord avec d'autres auteurs, que c'est surtout en été et au début de l'automne que le métabolisme de l'étang est au maximum. II y a donc un décalage entre les conditions optimum d'insolation (mai/juin) et de température (juillet/août) par rapport à la période où le métabolisme de l'étang est plus élevé (août/septembre), qui s'explique par la faible densité des peuplements phytoplanctoniques au printemps. A cette période, l'étang n'est donc pas prêt à exploiter les ressources lumineuses et caloriques du climat.

Du point de vue de la prévision des risques de désoxygénation dans les étangs de pisciculture, il apparaît donc que c'est en aoút/septembre que les risques sont les plus importants. En effet, à cette période, les fortes variations 
journalières de la teneur en oxygène créent des conditions écologiques peu favorables à la vie du poisson rendant l'écosystème étang instable (surtout dans le cas d'étangs de pisciculture). Des événements climatiques comme une baisse subite de la température ou des précipitations peuvent alors perturber l'équilibre du milieu en provoquant un brassage des eaux (remontée à la surface d'eaux désoxygénées) (PAPST et al., 1980) ou la mortalité du phytoplancton dont la dégradation pourra être la cause d'une désoxygénation grave BOYD et al., 1975).

Cette étude constitue donc une première étape dans le bilan de deux années de mesures en continu de la teneur en oxygène dissous, et dans la suite de ces travaux, nous envisagerons l'étude approfondie, sur des périodes définies, de l'impact des conditions climatiques (température, insolation, vent, précipitations) sur l'évolution de la teneur en oxygène en étang.

\section{RÉFÉRENCES BIBLIOGRAPHIQUES}

BARICA J., MATHIAS J.A., 1979. Oxygen depletion and winterkill risk in small prairie lakes under extended ice cover. J. Fish. Res. Boand Can., 36, 980-986.

BARTHELEMY D., COUTURE A., 1990. Oxygénation des étangs. Rapport IRRARégion Rhóne-Alpes, 1.53.

BOYD C.E., 1982. Water quality management for pond fish cuiture. Elsevier ed., 1-318.

BOYD C.E., 1985. Chemical budgets for Channel Catfish ponds. Trans. Am. Fish. Soc., 114, 291-298.

BOYD C.E., PRATHER E.E., JOHNSON E., 1975. Sudden mortality of a massive phytoplankton bloom. Weed Sci., 23, 61-67.

BOYD C.E., ROMAIRE R.P., JOHNSON E. 1978. Predicting early morning dissolved oxygen concentration in Channel Cattish pond. Trans. Am. Fish. Soc., 107 (3). 484-492.

BOYD C.E., LICHTKOPPLER F., 1979. Water quality management in pond fish culture. International Center for Aquaculture, Auburn University. Research and development, 22, 1-30.

COUTURE A., 1988. Essai de modélisation de la chute nocturne de l'oxygène dissous en étang. Rapport technique de D.E.A., Université Lyon I, Université Catholique de Lyon, 1-34.

EDELINE E., LAMBERT G., BINET W., FATTICCIONi H., 1968. Etude des eaux de la Meuse. Chapitre II : Enregistrement en continu des eaux de la Meuse à Monsin. Cebedeau, 295-296, 322-324.

ELORANTA P., 1985. Relations between physical and chemical variables of water quality and phytoplancton in one eutrophic pond. Aqua. Fennica., 15 (1), 105-113.

GINOT Y., 1988. Suivi des paramètres physico-chimiques et essais d'aérateur dans les bassins a Chevrettes de la station INRA-IFREMER de KOUROU. Rapport INRA-Kourou et IFREMER, Cayenne, Guyane Fr., 1-15.

GOURIEROUX C., MONFORT A., 1977. Cours de séries temporelles. Economica Ed., $1-380$.

GRYGIEREK E., WASILEWSKA 8.E., 1979. Possibitités de régulation des biocénoses des étangs à lélevage des poissons. Bull. Cent. Etud. Rech. Sci. Biarritz, 12 (3), 441-452.

HUNDING C., 1973. Diel variation in oxygen production and uptake in a microbenthic lit- 
toral community of a nutrient-poor lake. Oikos, 24, 352-360.

KEPENYES J., VARADI L., 1984. Aeration and oxygenation in aquaculture. $F A O /$ UNDP A DCP/REP, 84-21, 473-507.

LINGEMAN R., 1980. Signal analysis of irradiation, temperature and primary production in a small artificial pond : 1 . The annual signal. Int. Revue ges. Hyrdrobiol., 65 (6), 753 776.

MARZOLF G.R., SAUNDERS G.W., 1984. Patterns of diel oxygen change in ponds of tropical India. Verh. Internat. Verein. Limnol., 22, $1722-1726$.

MATHIAS J.A., BARICA J., 1980. Factors controlling oxygen depletion in ice-covered lakes. Can. J. Fish. Aquat. Sci., 37, 185194.

OLAH J., ZSIGRI A., KINTZLY A.V., 1978. Primary production estimations in fishponds by the mathematical evaluation of daily oxygen curves. Aquacultura Hungarica, 1 , 3-14.

PAPST M.H., MATHIAS J.A., BARICA J., 1980. Relationship between thermal stability and summer oxygen depletion in a prairie pothole Lake. Can. J. Fish. Aquat. Sci., 37, 1433-1438.

PEETERS J.C.H., EILERS P., 1978. The relationship between light intensity and photosynthesis. A simple mathematical model. Hydrobiol. Bull., 12, 134-136.

PELLETIER J.P., 1983. Mesure de la production primaire en milieu aquatique : problèmes méthodologiques et éléments d'interprétation. Revue Française des Sciences de l'Eau, 2, 339-366.

ROMAIRE R.P., BOYD C.E., 1978. Predicting nighttime oxygen depletion in Catfish ponds. Bull. Agr. Exp. Station, Auburn, Alabama, 505, 1-32.

SCHROEDER G.L., 1975. Nighttime Materiel Balance for Oxygen in fish ponds receiving organic wastes. Bamidgeh, 27, 65-74.

SCHROEDER G., BERNER-SAMSONOV E., 1986. The pond ecosystem and its control. In aquaculture of Cyprinids, INRA Paris, Billard et Marcel Ed., 243-254.

SIN A.W.C., CHIU M.T.L., 1982. SUmmer and winter kills in fish ponds of Hong-Kong and their possible prediction. Aquaculture, 29, 125-135. 